\title{
Implicit, Time-Dependent Variable Grid Finite Difference Methods for the Approximation of a Linear Waterflood
}

\author{
By Jim Douglas, Jr. and Mary Fanett Wheeler
}

\begin{abstract}
An implicit, time-dependent variable grid finite difference method based on the addition of an artificial diffusivity is introduced and analyzed for approximating the solution of a scalar conservation law in a single space variable. No relation between the grids at successive time steps is required for convergence. Two adaptive grid selection procedures are shown to be covered by the analysis. Analogous results are also established for an implicit upwinding procedure.
\end{abstract}

1. Introduction. The two-phase, incompressible, immiscible flow of water and oil in a homogeneous, linear (i.e., single space variable) porous medium can be described by the solution of the scalar conservation law

$$
\frac{\partial s}{\partial t}+\frac{\partial f(s)}{\partial x}=0,
$$

provided that capillary effects are ignored. This equation is derived from Darcy's law and the conservation of mass as follows. The subscripts " $o$ " and " $w$ " refer to oil and water, respectively. The symbols $\phi, k, \mu, \rho, q, p$, and $s$ represent porosity, permeability, viscosity, density, velocity, pressure and saturation, respectively, with $\phi$ being a rock property (constant by the assumption of homogeneity) and $k_{o}=$ $k(x) k_{r o}\left(s_{o}\right)$ being the product of the gross permeability $k(x)$, again a constant, and the relative permeability $k_{r o}\left(s_{o}\right)$ of the oil phase. The reservoir can be inclined; it will be assumed to have height $Z(x)$ linear in $x ; g$ is a gravitational constant. Then Darcy's law states that

$$
q_{o}=\frac{k_{o}}{\mu_{o}}\left(\frac{\partial p_{o}}{\partial x}+\rho_{o} g Z^{\prime}\right), \quad q_{w}=-\frac{k_{w}}{\mu_{w}}\left(\frac{\partial p_{w}}{\partial x}+\rho_{w} g Z^{\prime}\right) .
$$

Conservation of mass for the incompressible fluids requires that

$$
\phi \frac{\partial s_{o}}{\partial t}=-\frac{\partial q_{0}}{\partial x}, \quad \phi \frac{\partial s_{w}}{\partial t}=-\frac{\partial q_{w}}{\partial x} .
$$

If the medium is completely saturated by the water and oil, then $s_{o}+s_{w}=1$; set $s=s_{w}=1-s_{o}$. It then follows that $q_{o}+q_{w}$ is independent of $x$; for simplicity assume that the total flow rate is independent of time as well:

$$
q_{o}+q_{w}=Q, \text { a constant. }
$$

Received December 7, 1981; revised April 30, 1982

1980 Mathematics Subject Classification. Primary 65M10. 
Manipulation of (1.2)-(1.4) leads to the equation

$$
\phi \frac{\partial s}{\partial t}+\frac{\partial}{\partial x}\left[\frac{Q k_{w} / \mu_{w}}{k_{w} / \mu_{w}+k_{o} / \mu_{o}}\left(1-\frac{\left(\rho_{w}-\rho_{o}\right) g Z^{\prime}}{Q} \frac{k_{o}}{\mu_{o}}\right)\right]=0,
$$

which can be put into the form (1.1) by first changing the time variable by $t \rightarrow Q t / \phi$ and then setting

$$
f(s)=\frac{k_{w} / \mu_{w}}{k_{w} / \mu_{w}+k_{o} / \mu_{o}}\left[1-\frac{\left(\rho_{w}-\rho_{o}\right) g Z^{\prime}}{Q} \frac{k_{o}}{\mu_{o}}\right] .
$$

To specify a particular problem let the reservoir be the line $\mathbf{R}$ and assume the initial condition

$$
s(x, 0)=s_{0}(x), \quad x \in \mathbf{R},
$$

where for physical relevance $0 \leqslant s_{0}(x) \leqslant 1$. The function $f(s)$ is such that $f(0)=$ $f^{\prime}(0)=f^{\prime}(1)=0$ and $f(1)=1$; since $s_{0}(x)$ lies in the interval $[0,1]$, it follows that $s(x, t)$ is so bounded for all $x$ and $t$. It is expected that the physical solution will exhibit shocks, and the numerical procedure, if it is to be reliable, must be shown to give convergence to the correct discontinuous solution.

An implicit finite difference method based on the regularization of (1.1) by the addition of an artificial diffusivity depending on the function $f(s)$ and the time-dependent, variable spacing grid (a "vanishing viscosity" scheme) will be described and analyzed. The analysis follows the general outline presented by A. Y. Le Roux [5]; it also generalizes the analysis given by one of the authors [1] for the uniform, fixed grid case and presents the proofs for the results described by the other author [6] in her talk at a conference in Rio de Janeiro. The analysis is also extended to cover an implicit upwinding scheme.

Our principal theorem demonstrates that essentially any reasonable self-adaptive mesh selection process leads to convergence as the grid is refined. In particular, the method proposed by the authors and their colleagues [2] does give demonstrably convergent approximation for the examples treated in that paper.

2. The Finite Difference Procedure. Let $\Delta t>0$ and set $t^{n}=n \Delta t$. (It is an easy generalization to consider a variable time step; however, for notational convenience, we shall treat a constant time step.) Let $\delta^{n}=\left\{\ldots, x_{-1}^{n}, x_{0}^{n}, x_{1}^{n}, \ldots\right\}$, where $x_{i}^{n}-x_{i-1}^{n}$ $=h_{i}^{n}>0$, and $x_{ \pm i}^{n} \rightarrow \pm \infty$ as $i \rightarrow \infty$, be a possibly time-dependent grid. A centered finite difference analogue of (1.1) that conserves mass can be derived by using standard differencing for $f(s)_{x}$ and for the artificial diffusivity term and by approximating $s_{t}$ as follows. Denote the finite difference approximation at $\left(x_{i}^{n}, t^{n}\right)$ by $w_{i}^{n}$. Let $x_{i+1 / 2}^{n}=\frac{1}{2}\left(x_{i}^{n}+x_{i+1}^{n}\right)$, and extend the knot values $\left\{w_{i}^{n-1}\right\}$ to a function $w^{n-1}(x)$ by

$$
w^{n-1}(x)=w_{i}^{n-1}, \quad x_{i-1 / 2}^{n-1} \leqslant x<x_{i+1 / 2}^{n-1}
$$

Set

$$
\bar{w}_{i}^{n-1}=\frac{2}{h_{i}^{n}+h_{i+1}^{n}} \int_{x_{i-1 / 2}^{n}}^{x_{i+1 / 2}^{n} w^{n-1}(x) d x}
$$

i.e., $\bar{w}_{i}^{n-1}$ is the average value of $w^{n-1}$ over the interval $\left[x_{i-1 / 2}^{n}, x_{i+1 / 2}^{n}\right]$. For $n-1=0$ replace $w^{0}(x)$ by the initial values $s_{0}(x)$ in the evaluation of $\bar{w}_{i}^{0}$. 
Our difference equation is given by

$$
\begin{aligned}
\frac{w_{i}^{n}-\bar{w}_{i}^{n-1}}{\Delta t}+\frac{f\left(w_{i+1}^{n}\right)-f\left(w_{i-1}^{n}\right)}{h_{i}^{n}+h_{i+1}^{n}} & \\
-\frac{g_{i+1 / 2}^{n}\left(w_{i+1}^{n}-w_{i}^{n}\right)-g_{i-1 / 2}^{n}\left(w_{i}^{n}-w_{i-1}^{n}\right)}{h_{i}^{n}+h_{i+1}^{n}} & =0 .
\end{aligned}
$$

Note that the artificial diffusivity has been scaled locally to include the spatial increments as a factor; if $G_{i-1 / 2}^{n}=h_{i}^{n} g_{i-1 / 2}^{n}$, then the added term is a standard differencing for $-\left(G^{n} s_{x}^{n}\right)_{x}$. The requirements for the selection of $g_{i+1 / 2}^{n}$ will be given below, but essentially $g_{i+1 / 2}^{n}$ will approximate $f^{\prime}(s)$ for $s$ between $w_{i}^{n}$ and $w_{i+1}^{n}$.

3. Convergence Analysis. Our object is to establish the convergence of the approximate solution to the physical solution [3], [4] of $(1.1)$ in $L^{\infty}\left(0, T ; L^{1}(\mathbf{R})\right)$ as

$$
\Delta t+\max _{i, n} h_{i}^{n} \rightarrow 0 \text {. }
$$

Assume that

$$
\begin{aligned}
& \text { (a) } s_{0} \in L^{1}(\mathbf{R}) \cap B V(\mathbf{R}), \\
& \text { (b) } \operatorname{supp}\left(s_{0}\right) \subset \subset \mathbf{R} .
\end{aligned}
$$

Also, assume that $f$ is Lipschitz continuous on $\mathbf{R}$ with Lipschitz constant $F$.

Note that

$$
f\left(w_{i+1}^{n}\right)-f\left(w_{i}^{n}\right)=F_{i+1 / 2}^{n}\left(w_{i+1}^{n}-w_{i}^{n}\right)
$$

where

$$
\left|F_{i+1 / 2}^{n}\right| \leqslant \sup \left|\frac{f(u)-f(v)}{u-v}\right| \leqslant F
$$

and $F_{i+1 / 2}^{n}$ belongs to the subdifferential $f^{\prime}(u)$ for some value $u \in\left[w_{i}^{n} \wedge w_{i+1}^{n}\right.$, $\left.w_{i}^{n} \vee w_{i+1}^{n}\right]$. Assume that

$$
\begin{aligned}
g_{i+1 / 2}^{n} & \geqslant \sup \left\{\left|\frac{f(\alpha)-f(\beta)}{\alpha-\beta}\right|: w_{i}^{n} \wedge w_{i+1}^{n} \leqslant \alpha, \beta \leqslant w_{i}^{n} \vee w_{i+1}^{n}\right\} \\
& \geqslant\left|F_{i+1 / 2}^{n}\right|, \quad-\infty<i<\infty, n \geqslant 1 .
\end{aligned}
$$

While (3.5) is implicit, it does not raise a practical difficulty in actual petroleum engineering applications, for $f^{\prime}$ is relatively smooth.

Denote the $l^{\infty}\left(\delta^{n}\right)$-norm by $|\cdot|_{\infty}$ and the $B V\left(\delta^{n}\right)$-norm by

$$
\operatorname{Var}(z)=\sum_{i}\left|z_{i}-z_{i-1}\right|=\sum_{i}\left|\frac{z_{i}-z_{i-1}}{h_{i}^{n}}\right| h_{i}^{n} .
$$

LEMMA 3.1. $\left|w^{n}\right|_{\infty} \leqslant\left|w^{n-1}\right|_{\infty} \leqslant \cdots \leqslant\left\|s_{0}\right\|_{L^{\infty}(\mathbf{R})}$.

Proof. Clearly, $\left|w^{0}\right|_{\infty} \leqslant\left\|s_{0}\right\|_{L^{\infty}}$, so that it is necessary only to show that $\left|w^{n}\right|_{\infty} \leqslant\left|w^{n-1}\right|_{\infty}$. The difference equation (2.3) can be rewritten in the form

$$
\frac{w_{i}^{n}-\bar{w}_{i}^{n-1}}{\Delta t}=\theta_{i}^{n}\left(w_{i+1}^{n}-w_{i}^{n}\right)+\psi_{i}^{n}\left(w_{i-1}^{n}-w_{i}^{n}\right),
$$


where

$$
\begin{aligned}
& \text { (a) } \theta_{i}^{n}=\left(h_{i}^{n}+h_{i+1}^{n}\right)^{-1}\left(g_{i+1 / 2}^{n}-F_{i+1 / 2}^{n}\right), \\
& \text { (b) } \psi_{i}^{n}=\left(h_{i}^{n}+h_{i+1}^{n}\right)^{-1}\left(g_{i-1 / 2}^{n}+F_{i+1 / 2}^{n}\right) .
\end{aligned}
$$

The constraint (3.5) implies that $\theta_{i}^{n}$ and $\psi_{i}^{n}$ are nonnegative, and the usual maximum principle argument shows that

$$
\inf _{j} \bar{w}_{j}^{n-1} \leqslant w_{i}^{n} \leqslant \sup _{j} \bar{w}_{j}^{n-1}
$$

Thus, it follows that

$$
\left|w^{n}\right|_{\infty} \leqslant\left|w^{n-1}\right|_{\infty}
$$

as was to be proved.

An obvious corollary of (3.8) is that, if $0 \leqslant s_{0}(x) \leqslant 1$, then $0 \leqslant w_{i}^{n} \leqslant 1$ for all $i$ and $n$.

LEMMA 3.2. $\operatorname{Var}\left(w^{n}\right) \leqslant \operatorname{Var}\left(w^{n-1}\right) \leqslant \cdots \leqslant \operatorname{Var}\left(s_{0}\right)$.

Proof. Set $z_{i}^{n}=w_{i+1}^{n}-w_{i}^{n}$ and $\bar{z}_{i}^{n-1}=\bar{w}_{i+1}^{n-1}-\bar{w}_{i}^{n-1}$. Then,

$$
\frac{z_{i}^{n}-\bar{z}_{i}^{n-1}}{\Delta t}=\theta_{i+1}^{n} z_{i+1}^{n}-\theta_{i}^{n} z_{i}^{n}-\psi_{i+1}^{n} z_{i}^{n}+\psi_{i}^{n} z_{i-1}^{n}
$$

or

$$
\left(\frac{1}{\Delta t}+\theta_{i}^{n}+\psi_{i+1}^{n}\right) z_{i}^{n}=\frac{1}{\Delta t} \bar{z}_{i}^{n-1}+\theta_{i+1}^{n} z_{i+1}^{n}+\psi_{i}^{n} z_{i-1}^{n} .
$$

Thus, since $\theta_{i}^{n} \geqslant 0$ and $\psi_{i}^{n} \geqslant 0$,

$$
\sum_{i}\left[1+\left(\theta_{i}^{n}+\psi_{i+1}^{n}\right) \Delta t\right]\left|z_{i}^{n}\right| \leqslant \sum_{i}\left|\bar{z}_{i}^{n-1}\right|+\sum_{i}\left(\theta_{i}^{n}+\psi_{i+1}^{n}\right) \Delta t\left|z_{i}^{n}\right|,
$$

or

$$
\operatorname{Var}\left(w^{n}\right)=\sum_{i}\left|z_{i}^{n}\right| \leqslant \sum_{i}\left|\bar{z}_{i}^{n-1}\right| .
$$

Now, let $I_{j}=\left[x_{j-1 / 2}^{n}, x_{j+1 / 2}^{n}\right]$, and assume that $\alpha_{j}$ and $\beta_{j}$ are points in $I_{j}$ such that

$$
w^{n-1}\left(\alpha_{j}\right)=\max _{I_{j}} w^{n-1}(x), \quad w^{n-1}\left(\beta_{j}\right)=\min _{I_{j}} w^{n-1}(x),
$$

where $w^{n-1}$ is considered to be the multivalued function resulting from filling in its jumps. Let $\left\{\gamma_{k}\right\}$ be the ordered union of $\left\{\alpha_{j}\right\}$ and $\left\{\beta_{j}\right\}$. Then,

$$
\begin{aligned}
\sum_{i}\left|\bar{z}_{i}^{n-1}\right| & \leqslant \sum_{j} \max \left\{\left|w^{n-1}\left(\alpha_{j+1}\right)-w^{n-1}\left(\beta_{j}\right)\right|,\left|w^{n-1}\left(\beta_{j+1}\right)-w^{n-1}\left(\alpha_{j}\right)\right|\right\} \\
& \leqslant \sum_{k}\left|w^{n-1}\left(\gamma_{k+1}\right)-w^{n-1}\left(\gamma_{k}\right)\right| \leqslant \operatorname{Var}\left(w^{n-1}\right) .
\end{aligned}
$$

Thus, $\operatorname{Var}\left(w^{n}\right) \leqslant \operatorname{Var}\left(w^{n-1}\right)$ for $n \geqslant 1$. Clearly, $\operatorname{Var}\left(w^{0}\right) \leqslant \operatorname{Var}\left(s_{0}\right)$, and the lemma is proved.

An immediate consequence of Lemma 3.2 is that the $l^{1}(\mathbf{R})$-norm of the first $x$-difference (over $\delta^{n}$ ) of the approximate solution $w^{n}$ satisfies the inequality

$$
\left|\partial_{x} w^{n}\right|_{1, n}=\sum_{i}\left|\frac{w_{i}^{n}-w_{i-1}^{n}}{h_{i}^{n}}\right| h_{i}^{n} \leqslant\left|\partial_{x} w^{n-1}\right|_{1, n-1} \leqslant \cdots \leqslant \operatorname{Var}\left(s_{0}\right) .
$$


Another sum is also useful as an alternate $l^{1}(\mathbf{R})$-norm. Let

$$
|u|_{1, \tilde{n}}=\sum_{i}\left|u_{i}\right| \frac{h_{i}^{n}+h_{i+1}^{n}}{2}
$$

LEMMA 3.3. Let $\partial_{t} w_{i}^{n}=\left(w_{i}^{n}-\bar{w}_{i}^{n-1}\right) / \Delta t$, and assume that $\left|F_{i+1 / 2}^{n}\right| \leqslant g_{i+1 / 2}^{n} \leqslant F$. Then,

$$
\left|\partial_{t} w^{n}\right|_{1, \tilde{n}}=\sum_{i}\left|\partial_{t} w_{i}^{n}\right| \frac{h_{i}^{n}+h_{i+1}^{n}}{2} \leqslant 2 F\left|\partial_{x} w^{n}\right|_{1, n} \leqslant 2 F \operatorname{Var}\left(s_{0}\right)
$$

Proof. A slight rearrangement of (3.6) using (3.7) leads to

$$
\left(h_{i}^{n}+h_{i+1}^{n}\right) \partial_{t} w_{i}^{n}=\left(g_{i+1 / 2}^{n}-F_{i+1 / 2}^{n}\right) z_{i}^{n}-\left(g_{i-1 / 2}^{n}+F_{i-1 / 2}^{n}\right) z_{i-1}^{n}
$$

so

$$
\frac{1}{2}\left(h_{i}^{n}+h_{i+1}^{n}\right)\left|\partial_{t} w_{i}^{n}\right| \leqslant F\left(\left|z_{i}^{n}\right|+\left|z_{i-1}^{n}\right|\right),
$$

from which (3.10) follows.

LEMMA 3.4. $\left|w^{n}\right|_{1, \tilde{n}} \leqslant\left\|s_{0}\right\|_{L^{\prime}(\mathbf{R})}+2 F t^{n} \operatorname{Var}\left(s_{0}\right)$.

Proof. Since

$$
\begin{gathered}
\left|\bar{w}_{i}^{n-1}\right| \leqslant \frac{2}{h_{i}^{n}+h_{i+1}^{n}} \int_{x_{i-1 / 2}^{n}}^{x_{i+1 / 2}^{n}}\left|w^{n-1}(x)\right| d x, \\
\sum_{i}\left|\bar{w}_{i}^{n-1}\right| \frac{h_{i}^{n}+h_{i+1}^{n}}{2} \leqslant \int\left|w^{n-1}\right| d x=\sum_{j}\left|w_{j}^{n-1}\right| \frac{h_{j}^{n-1}+h_{j+1}^{n-1}}{2} .
\end{gathered}
$$

Thus,

$$
\begin{aligned}
& \sum_{i}\left|w_{i}^{n}\right| \frac{h_{i}^{n}+h_{i+1}^{n}}{2} \leqslant \sum_{i}\left|\bar{w}_{i}^{n-1}\right| \frac{h_{i}^{n}+h_{i+1}^{n}}{2}+F \Delta t \sum_{i}\left(\left|z_{i}^{n}\right|+\left|z_{i-1}^{n}\right|\right) \\
& \quad \leqslant \sum_{j}\left|w_{j}^{n-1}\right| \frac{h_{j}^{n-1}+h_{j+1}^{n-1}}{2}+2 F \Delta t \operatorname{Var}\left(w^{n}\right)
\end{aligned}
$$

from which the lemma follows easily.

Consider now a family of approximate solutions. Let $\tau_{k}=\left\{\Delta t_{k} ; \delta_{k}^{n}, n=0,1, \ldots\right\}$, where $\delta_{k}^{n}=\left\{x_{i, k}^{n}\right\}$, and let $w_{i, k}^{n}$ denote the solution of (2.3) related to $\tau_{k}$. Assume that

$$
\max _{i, n} h_{i, k}^{n} \leqslant K \Delta t_{k} \rightarrow 0
$$

as $k \rightarrow \infty$. (This constraint is rather unimportant, as it is the ability to use small $h$ locally that is of interest; also, (3.13) is not required if $\delta_{k}^{n}$ never depends on $n$.) Associate a function $w_{k}(x, t)$ with the knot values $w_{i, k}^{n}$ by first extending $\left\{w_{i, k}^{n}, i=\right.$ $0, \pm 1, \ldots\}$ be piecewise-linear interpolation in $x$ to $\hat{w}_{k}^{n}(x)$ and then $\left\{\hat{w}_{k}^{n}, n=0,1, \ldots\right\}$ by piecewise-linear interpolation in $t$. Lemmas $3.1-3.4$ have the following interpretations in terms of $w_{k}$. 
LEMMA 3.5. Under the hypothesis (3.13), the function $w_{k}(x, t)$ satisfies the following estimates:
(a) $\left\|w_{k}\right\|_{L^{\infty}(\mathbf{R} \times[0, T])} \leqslant\left\|s_{0}\right\|_{L^{\infty}(\mathbf{R})}$,
(b) $\left\|w_{k}(t)\right\|_{L^{\prime}(\mathbf{R})} \leqslant\left\|s_{0}\right\|_{L^{\prime}(\mathbf{R})}+2 F t \operatorname{Var}\left(s_{0}\right)$,
(c) $\left\|\frac{\partial w_{k}}{\partial x}\right\|_{L^{\infty}\left(0, T ; L^{\prime}(\mathbf{R})\right)} \leqslant \operatorname{Var}\left(s_{0}\right)$,
(d) $\left\|\frac{\partial w_{k}}{\partial t}\right\|_{L^{\infty}\left(0, T ; L^{\prime}(\mathbf{R})\right)} \leqslant(2 F+7 K) \operatorname{Var}\left(s_{0}\right)$.

Proof. The inequalities (3.14a) and (3.14c) are immediate; (3.14b) results from noting the trivial inequality

$$
\left\|\hat{w}_{k}^{n}\right\|_{L^{\prime}(\mathbf{R})} \leqslant\left\|w_{k}^{n}\right\|_{L^{\prime}(\mathbf{R})} .
$$

The estimate for $\partial w_{k} / \partial t$ requires argument and is the source of the restriction (3.13). Let $x \in\left[x_{i-1 / 2}^{n}, x_{i+1 / 2}^{n}\right]$. With $w(x, t)=w_{k}(x, t)$, and $t^{n-1}<t<t^{n}$,

$$
\begin{aligned}
\frac{\partial w}{\partial t}(x, t) & =\frac{\hat{w}^{n}(x)-\hat{w}^{n-1}(x)}{\Delta t} \\
& =\frac{w_{i}^{n}-\bar{w}_{i}^{n-1}}{\Delta t}+\frac{1}{\Delta t} \int_{x_{i}^{n}}^{x} \frac{\partial \hat{w}^{n}}{\partial x} d \xi+\frac{\bar{w}_{i}^{n-1}-\hat{w}^{n-1}(x)}{\Delta t} .
\end{aligned}
$$

First, (3.10) implies that

$$
\sum_{i}\left|\frac{w_{i}^{n}-\bar{w}_{i}^{n-1}}{\Delta t}\right| \frac{h_{i}^{n}+h_{i+1}^{n}}{2} \leqslant 2 F \operatorname{Var}\left(s_{0}\right) .
$$

Next, by (3.13) and (3.14c),

$$
\sum_{i} \int_{x_{i-1 / 2}^{n}}^{x_{i+1 / 2}^{n}} d x\left|\int_{x_{i}^{n}}^{x} \frac{\partial \hat{w}^{n}}{\partial x} d x\right| \leqslant \sum_{i} \frac{h_{i}^{n}+h_{i}^{n+1}}{2 \Delta t} \int_{x_{i-1 / 2}^{n}}^{x_{i+1 / 2}^{n}}\left|\frac{\partial \hat{w}^{n}}{\partial x}\right| d x \leqslant K \operatorname{Var}\left(s_{0}\right) .
$$

Essentially two cases arise in the treatment of the third term. If there exists $\bar{y}_{i}^{n} \in\left[x_{i-1 / 2}^{n}, x_{i+1 / 2}^{n}\right]$ such that $\hat{w}_{i}^{n-1}\left(\bar{y}_{i}^{n}\right)=\bar{w}_{i}^{n-1}$, then

$$
\bar{w}_{i}^{n-1}-\hat{w}^{n-1}(x)=-\int_{\bar{y}_{i}^{n}}^{x} \frac{\partial \hat{w}^{n-1}}{\partial x} d \xi,
$$

and

$$
\begin{aligned}
\int_{x_{i-1 / 2}^{n}}^{x_{i+1 / 2}^{n}}\left|\frac{\bar{w}_{i}^{n-1}-\hat{w}^{n-1}(x)}{\Delta t}\right| d x & \leqslant \frac{h_{i}^{n}+h_{i+1}^{n}}{2 \Delta t} \int_{x_{i-1 / 2}^{n}}^{x_{i+1 / 2}^{n}}\left|\frac{\partial \hat{w}^{n-1}}{\partial x}\right| d \xi \\
& \leqslant K \operatorname{Var}_{\left[x_{i-1 / 2}^{n}, x_{i+1 / 2}^{n}\right]}\left(\hat{w}^{n-1}\right) .
\end{aligned}
$$

If no such $\bar{y}_{i}^{n}$ exists, assume that $w^{n-1}(x)<\bar{w}_{i}^{n-1}$ on $\left[x_{i-1 / 2}^{n}, x_{i+1 / 2}^{n}\right]=I_{i}^{n}$. With $w^{n-1}(x)=w_{j}^{n-1}$ on $I_{j}^{n-1}$ as before, we consider first the case that $w^{n-1}$ assumes at least two values on $I_{i}^{n}$. Then, $\hat{w}^{n-1}$ must assume a value between the two some place on $I_{i}^{n}$; hence, there exists $\tilde{y}_{i}^{n} \in I_{i}^{n}$ such that

$$
\bar{w}_{i}^{n-1}-\hat{w}^{n-1}\left(\tilde{y}_{i}^{n}\right) \leqslant \operatorname{Var}_{I_{i}^{n}}\left(w^{n-1}\right) .
$$


The argument above then leads to the inequality

$$
\int_{I_{i}^{n}}\left|\frac{\bar{w}_{i}^{n-1}-\hat{w}^{n-1}(x)}{\Delta t}\right| d x \leqslant K\left(\operatorname{Var}_{I_{i}^{n}}\left(w^{n-1}\right)+\operatorname{Var}_{I_{i}^{n}}\left(\hat{w}^{n-1}\right)\right) .
$$

Finally, we are left with the case for which $w^{n-1}(x)=\bar{w}_{i}^{n-1}$ on $I_{i}^{n}$. Let $J$ indicate the union of consecutive intervals $I_{i_{1}}^{n}, I_{i_{1}+1}^{n}, \ldots, I_{i_{2}}^{n}$, where $i_{1} \leqslant i \leqslant i_{2}$, on which $w^{n-1}(x)$ remains constant and equal to $\bar{w}_{i}^{n-1}$ and on which $w^{n-1}(x)<\bar{w}_{i}^{n-1}$. If $J^{*}=I_{i_{1}-1}^{n} \cup$ $J \cup I_{i_{2}+1}^{n}$, then it follows that

$$
\bar{w}_{i}^{n-1}-\hat{w}^{n-1}\left(x_{i_{2}+1 / 2}^{n}\right) \leqslant \operatorname{Var}_{J^{*}}\left(w^{n-1}\right)
$$

and

$$
\int_{J}\left|\frac{\bar{w}_{i}^{n-1}-\hat{w}^{n-1}(x)}{\Delta t}\right| d x \leqslant \frac{|J|}{\Delta t}\left(\operatorname{Var}_{J^{*}}\left(w^{n-1}\right)+\operatorname{Var}_{J^{*}}\left(\hat{w}^{n-1}\right)\right) .
$$

The constancy of $w^{n-1}$ on $J$ and the nonexistence of a point at which $\hat{w}^{n-1}$ equals $\bar{w}_{i}^{n-1}$ implies that $|J| \leqslant K \Delta t$. Hence

$$
\int_{J}\left|\frac{\bar{w}_{i}^{n-1}-\hat{w}^{n-1}(x)}{\Delta t}\right| d x \leqslant K\left(\operatorname{Var}_{J^{*}}\left(w^{n-1}\right)+\operatorname{Var}_{J^{*}}\left(\hat{w}^{n-1}\right)\right) .
$$

The variation of $w^{n-1}$ or $\hat{w}^{n-1}$ over some interval $I_{i}^{n}$ occurs at most three times as a result of the decomposition into cases above. Thus,

$$
\begin{gathered}
\sum_{i} \int_{I_{i}^{n}}\left|\frac{\bar{w}_{i}^{n-1}-\hat{w}^{n-1}(x)}{\Delta t}\right| d x \\
\quad \leqslant(2 F+K) \operatorname{Var}\left(s_{0}\right)+3 K\left(\operatorname{Var}\left(w^{n-1}\right)+\operatorname{Var}\left(\hat{w}^{n-1}\right)\right) \\
\leqslant(2 F+7 K) \operatorname{Var}\left(s_{0}\right) .
\end{gathered}
$$

Note that, if $\delta^{n}$ were constant, $\bar{w}_{i}^{n-1}=w_{i}^{n-1}$ and no perturbation terms would arise, so that (3.13) would be unnecessary.

Standard functional analysis implies that a subsequence, say $\left\{w_{k_{m}}\right\}$, of $\left\{w_{k}\right\}$ converges in $L^{\infty}\left(0, T ; L^{1}(\mathbf{R})\right)$ to a function $w \in L^{\infty}\left(0, T ; L^{1}(\mathbf{R})\right)$. The first objective is to show that $w$ is a weak solution of (1.1), (1.7); i.e., if $\varphi \in C_{0}^{\infty}(\mathbf{R} \times[0, T))$, then

$$
\int_{0}^{T} \int_{\mathbf{R}}\left(w \varphi_{t}+f(w) \varphi_{x}\right) d x d t+\int_{\mathbf{R}} s_{0}(x) \varphi(x, 0) d x=0 .
$$

Afterwards, it will be shown that $w$ is the unique weak solution satisfying the Kružkov criterion for the physically relevant solution; hence, the entire sequence $\left\{w_{k}\right\}$ will converge to the desired weak solution.

It is convenient to define an alternate, piecewise-constant extension of $w_{i, k}^{n}$. Let $\tilde{w}_{k}(x, t)=w_{i, k}^{n}$ on $\left[x_{i-1 / 2, k}^{n}, x_{i+1 / 2, k}^{n}\right) \times\left[t_{k}^{n}, t_{k}^{n+1}\right)$. Note that, if $w_{k_{m}} \rightarrow w$ in $L^{\infty}\left(0, T ; L^{1}(\mathbf{R})\right)$, then so does $\left\{\tilde{w}_{k_{m}}\right\}$. Now, let $\varphi \in C_{0}^{\infty}(\mathbf{R} \times[0, T))$ and multiply Eq. (2.3) for $w_{i}^{n}=w_{i, k_{m}}^{n}$ by

$$
\varphi_{i}^{n}\left(h_{i}^{n}+h_{i+1}^{n}\right) \Delta t / 2=\varphi_{i, k_{m}}^{n}\left(h_{i, k_{m}}^{n}+h_{i+1, k_{m}}^{n}\right) \Delta t_{k_{m}} / 2
$$


and add on all $i$ and $n \geqslant 1$. The individual terms can be treated as follows. First, by (3.10),

$$
\begin{aligned}
\sum_{i} \frac{w_{i}^{n}-\bar{w}_{i}^{n-1}}{\Delta t} \varphi_{i}^{n} & \frac{h_{i}^{n}+h_{i+1}^{n}}{2} \\
& =\sum_{i} \int_{x_{i-1 / 2}^{n}}^{x_{i+1 / 2}^{n}} \frac{w^{n}(x)-\bar{w}_{i}^{n-1}}{\Delta t} \varphi\left(x, t^{n}\right) d x+O\left(h \operatorname{Var}\left(s_{0}\right)\right)
\end{aligned}
$$

where

$$
h=\sup _{i, n} h_{i}^{n}
$$

Recall that

$$
\int_{x_{i-1 / 2}^{n}}^{x_{i+1 / 2}^{n}}\left(w^{n-1}(x)-\bar{w}_{i}^{n-1}\right) d x=0,
$$

and set $\bar{\varphi}_{i}^{n}$ equal to the average value of $\varphi\left(x, t^{n}\right)$ over $\left[x_{i-1 / 2}^{n}, x_{i+1 / 2}^{n}\right]$. Then,

$$
\begin{aligned}
& \int_{x_{i-1 / 2}^{n}}^{x_{i+1 / 2}^{n}} \bar{w}_{i}^{n-1} \varphi\left(x, t^{n}\right) d x \\
& \quad=\int_{x_{i-1 / 2}^{n}}^{x_{i+1 / 2}^{n}}\left[w^{n-1}(x) \varphi\left(x, t^{n}\right)+\left(\bar{w}_{i}^{n-1}-w^{n-1}(x)\right)\left(\varphi\left(x, t^{n}\right)-\bar{\varphi}_{i}^{n}\right)\right] d x \\
& \quad=\int_{x_{i-1 / 2}^{n}}^{x_{i+1 / 2}^{n}} w^{n-1}(x) \varphi\left(x, t^{n}\right) d x+O\left(\left(h_{i}^{n}+h_{i+1}^{n}\right)^{2} \operatorname{Var}_{\left[x_{i-1 / 2}^{n}, x_{i+1 / 2}^{n}\right]}\left(w^{n-1}\right)\right),
\end{aligned}
$$

since $\left|\bar{w}_{i}^{n-1}-w^{n-1}(x)\right| \leqslant \operatorname{Var}_{\left[x_{i-1 / 2}^{n}, x_{i+1 / 2}^{n}\right]}\left(w^{n-1}\right)$ on the subinterval. Thus, (3.17) can be changed to read

$$
\sum_{i} \frac{w_{i}^{n}-\bar{w}_{i}^{n-1}}{\Delta t} \varphi_{i}^{n} \frac{h_{i}^{n}+h_{i+1}^{n}}{2}=\int \frac{w^{n}-w^{n-1}}{\Delta t} \varphi^{n} d x+O\left(h \operatorname{Var}\left(s_{0}\right)\right)
$$

using the restriction (3.13). Add on time:

$$
\begin{aligned}
& \sum_{n=1}^{\infty} \sum_{i} \frac{w_{i}^{n}-\bar{w}_{i}^{n-1}}{\Delta t} \varphi_{i}^{n} \frac{h_{i}^{n}+h_{i+1}^{n}}{2} \Delta t \\
&=\sum_{n=1}^{\infty} \Delta t \int \frac{w^{n}-w^{n-1}}{\Delta t} \varphi^{n} d x+O\left(h \operatorname{Var}\left(s_{0}\right)\right) \\
&=-\int w^{0} \varphi^{1} d x-\sum_{n=1}^{\infty} \Delta t \int w^{n} \frac{\varphi^{n+1}-\varphi^{n}}{\Delta t} d x+O\left(h \operatorname{Var}\left(s_{0}\right)\right) \\
&=-\int s_{0}(x) \varphi(x, 0) d x-\int_{0}^{T} \int \tilde{w} \frac{\partial \varphi}{\partial t} d x d t \\
&+O\left((\Delta t+h)\left(\operatorname{Var}\left(s_{0}\right)+\left\|s_{0}\right\|_{L^{1}(\mathbf{R})}\right)\right) \\
& \rightarrow-\int s_{0}(x) \varphi(x, 0) d x-\int_{0}^{T} \int w \frac{\partial \varphi}{\partial t} d x d t
\end{aligned}
$$

as $k_{m} \rightarrow \infty$, where $w$ is the limit of $\left\{\tilde{w}_{k_{m}}\right\}$. 
Next,

$$
\begin{aligned}
\sum_{n=1}^{\infty} \sum_{i} \frac{f\left(w_{i+1}^{n}\right)-f\left(w_{i-1}^{n}\right)}{h_{i}^{n}+} h_{i+1}^{n} & \varphi_{i}^{n} \frac{h_{i}^{n}+h_{i+1}^{n}}{2} \Delta t \\
& =-\sum_{n=1}^{\infty} \sum_{i} f\left(w_{i}^{n}\right) \frac{\varphi_{i+1}^{n}-\varphi_{i-1}^{n}}{h_{i}^{n}+h_{i+1}^{n}} \frac{h_{i}^{n}+h_{i+1}^{n}}{2} \Delta t \\
& \rightarrow-\int_{0}^{T} \int f(w) \frac{\partial \varphi}{\partial x} d x d t
\end{aligned}
$$

by the Lipschitz continuity of $f$. Finally,

$$
\begin{aligned}
\mid \sum_{n=1}^{\infty} \sum_{i} & \frac{g_{i+1 / 2}^{n}\left(w_{i+1}^{n}-w_{i}^{n}\right)-g_{i-1 / 2}^{n}\left(w_{i}^{n}-w_{i-1}^{n}\right)}{h_{i}^{n}+h_{i+1}^{n}} \varphi_{i}^{n} \frac{h_{i}^{n}+h_{i+1}^{n}}{2} \Delta t \mid \\
& =\left|\frac{1}{2} \sum_{n=1}^{\infty} \sum_{i} g_{i-1 / 2}^{n} \frac{w_{i}^{n}-w_{i-1}^{n}}{h_{i}^{n}} \frac{\varphi_{i}^{n}-\varphi_{i-1}^{n}}{h_{i}^{n}}\left(h_{i}^{n}\right)^{2} \Delta t\right| \\
& =O\left(h \operatorname{Var}\left(s_{0}\right)\right),
\end{aligned}
$$

by Lemma 3.2. The combination of (3.22), (3.23), and (3.24) leads to the conclusion that the limit function $w$ satisfies (3.16); consequently, $w$ is a weak solution of the initial value problem (1.1), (1.7).

It remains to show that the limit function $w$ is unique, that the entire sequence $\left\{w_{k}\right\}$ converges to $w$ in $L^{\infty}\left(0, T ; L^{1}(\mathbf{R})\right)$, and that it is the physically relevant weak solution. This will be done by showing that $w$ satisfies the two conditions of Kružkov [3] that guarantee that it is the physically relevant weak solution. The simpler of the two conditions is to prove that

$$
\lim _{\substack{t \downarrow 0 \\ t \notin E}}\left\|w(\cdot, t)-s_{0}\right\|_{L^{\prime}(\mathbf{R})}=0,
$$

where the exceptional set $E$ must have measure zero. The difference $w(x, t)-s_{0}(x)$ can be represented in the form

$$
\begin{aligned}
w(x, t)-s_{0}(x)= & \left(w-w_{k_{m}}\right)(x, t)+\int_{0}^{t} \frac{\partial w_{k_{m}}}{\partial t}(x, \tau) d \tau \\
& +\left(w_{k_{m}}(x, 0)-s_{0}(x)\right) .
\end{aligned}
$$

The third term tends to zero in $L^{1}(\mathbf{R})$ by the construction of $w_{k_{m}}(x, 0)$. The integral is $O(t)$ in $L^{1}(\mathbf{R})$, by Lemma 3.5. The first term tends to zero in $L^{1}(\mathbf{R})$ for almost all $t$, by Fubini. Consequently, (3.25) holds.

The more difficult condition to establish is that, for $0 \leqslant \varphi \in C_{0}^{\infty}(\mathbf{R} \times(0, T))$ and $k \in \mathbf{R}$,

$$
\int_{0}^{T} \int\left\{|w-k| \varphi_{t}+\operatorname{sgn}(w-k)[f(w)-f(k)] \varphi_{x}\right\} d x d t \geqslant 0
$$

The lemma below and the argument given in (3.17)-(3.24) above will be used to demonstrate (3.27). 
LEMMA 3.6. Let $k \in \mathbf{R}$. Then,

$$
\begin{aligned}
& \frac{\left|w_{i}^{n}-k\right|-\left|\bar{w}_{i}^{n-1}-k\right|}{\Delta t} \\
& +\frac{\operatorname{sgn}\left(w_{i+1}^{n}-k\right)\left\{f\left(w_{i+1}^{n}\right)-f(k)\right\}-\operatorname{sgn}\left(w_{i-1}^{n}-k\right)\left\{f\left(w_{i-1}^{n}\right)-f(k)\right\}}{h_{i}^{n}+h_{i+1}^{n}} \\
& \leqslant \frac{g_{i+1 / 2}^{n}\left(\left|w_{i+1}^{n}-k\right|-\left|w_{i}^{n}-k\right|\right)-g_{i-1 / 2}^{n}\left(\left|w_{i}^{n}-k\right|-\left|w_{i-1}^{n}-k\right|\right)}{h_{i}^{n}+h_{i+1}^{n}}
\end{aligned}
$$

Proof. Set $\mu_{i}^{n}=\Delta t\left(h_{i}^{n}+h_{i+1}^{n}\right)^{-1}$. Add and subtract $k$ in the time-difference term and $f(k)$ in the transport term in (2.3), and then multiply by $\operatorname{sgn}\left(w_{i}^{n}-k\right)$ :

$$
\begin{aligned}
\left|w_{i}^{n}-k\right|=\operatorname{sgn}\left(w_{i}^{n}-k\right) \cdot\left(\bar{w}_{i}^{n-1}-k\right) \\
+\mu_{i}^{n}\left[\operatorname{sgn}\left(w_{i-1}^{n}-k\right)\left\{f\left(w_{i-1}^{n}\right)-f(k)\right\}\right. \\
\quad-\operatorname{sgn}\left(w_{i+1}^{n}-k\right)\left\{f\left(w_{i+1}^{n}\right)-f(k)\right\} \\
+g_{i+1 / 2}^{n}\left\{\left|w_{i+1}^{n}-k\right|-\left|w_{i}^{n}-k\right|\right\} \\
\left.\quad-g_{i-1 / 2}^{n}\left\{\left|w_{i}^{n}-k\right|-\left|w_{i-1}^{n}-k\right|\right\}+R_{i}^{n}\right],
\end{aligned}
$$

where, if $f\left(w_{j}^{n}\right)-f(k)=G_{j}^{n}\left(w_{j}^{n}-k\right)$,

$$
\begin{aligned}
R_{i}^{n}= & {\left[\operatorname{sgn}\left(w_{i}^{n}-k\right)-\operatorname{sgn}\left(w_{i-1}^{n}-k\right)\right]\left\{g_{i-1 / 2}^{n}+G_{i-1}^{n}\right\}\left(w_{i-1}^{n}-k\right) } \\
& +\left[\operatorname{sgn}\left(w_{i}^{n}-k\right)-\operatorname{sgn}\left(w_{i+1}^{n}-k\right)\right]\left\{g_{i+1 / 2}^{n}-G_{i+1}^{n}\right\}\left(w_{i+1}^{n}-k\right) .
\end{aligned}
$$

The first term on the right-hand side of (3.30) vanishes unless $k$ falls between $w_{i-1}^{n}$ and $w_{i}^{n}$. If $k$ does fall there, (3.5) implies that $g_{i-1 / 2}^{n}+G_{i-1}^{n} \geqslant 0$, and the product is nonpositive. Similarly, the other term is also nonpositive. Hence,

$$
R_{i}^{n} \leqslant 0
$$

and (3.28) is valid.

Let $\varphi \geqslant 0, \varphi \in C_{0}^{\infty}(\mathbf{R} \times(0, T))$. Multiply (3.28) by $\varphi_{i}^{n}\left(h_{i}^{n}+h_{i+1}^{n}\right) \Delta t / 2$ and sum over $i$ and $n, n \geqslant 1$. The analogues of (3.23) and (3.24) are

$$
\begin{aligned}
& \sum_{n=1}^{\infty} \sum_{i} \frac{\operatorname{sgn}\left(w_{i+1}^{n}-k\right)\left\{f\left(w_{i+1}^{n}\right)-f(k)\right\}-\operatorname{sgn}\left(w_{i-1}^{n}-k\right)\left\{f\left(w_{i-1}^{n}\right)-f(k)\right\}}{h_{i}^{n}+h_{i+1}^{n}} \\
& \times \varphi_{i}^{n} \frac{h_{i}^{n}+h_{i+1}^{n}}{2} \Delta t \\
& =-\sum_{n=1}^{\infty} \sum_{i} \operatorname{sgn}\left(w_{i}^{n}-k\right)\left\{f\left(w_{i}^{n}\right)-f(k)\right\} \frac{\varphi_{i+1}^{n}-\varphi_{i-1}^{n}}{h_{i}^{n}+h_{i+1}^{n}} \frac{h_{i}^{n}+h_{i+1}^{n}}{2} \Delta t \\
& \rightarrow-\int_{0}^{T} \int \operatorname{sgn}(w-k)\{f(w)-f(k)\} \frac{\partial \varphi}{\partial x} d x d t
\end{aligned}
$$


and

$$
\begin{aligned}
& \mid \sum_{n=1}^{\infty} \sum_{i} \frac{g_{i+1 / 2}^{n}\left\{\left|w_{i+1}^{n}-k\right|-\left|w_{i}^{n}-k\right|\right\}-g_{i-1 / 2}^{n}\left\{\left|w_{i}^{n}-k\right|-\left|w_{i-1}^{n}-k\right|\right\}}{h_{i}^{n}+h_{i+1}^{n}} \\
& \quad \times \varphi_{i}^{n} \frac{h_{i}^{n}+h_{i+1}^{n}}{2} \Delta t \mid \\
& =\left|\frac{1}{2} \sum_{n=1}^{\infty} \sum_{i} g_{i-1 / 2}^{n} \frac{\left|w_{i}^{n}-k\right|-\left|w_{i-1}^{n}-k\right|}{h_{i}^{n}} \frac{\varphi_{i}^{n}-\varphi_{i-1}^{n}}{h_{i}^{n}}\left(h_{i}^{n}\right)^{2} \Delta t\right| \\
& =O\left(h \operatorname{Var}\left(s_{0}\right)\right) .
\end{aligned}
$$

Since $\varphi \geqslant 0$ and $\left|\bar{w}_{i}^{n-1}-k\right|$ is a constant, the orthogonality to constants expressed in (3.19) and used in (3.20) remains applicable. Consequently,

$$
\begin{aligned}
\sum_{n=1}^{\infty} \sum_{i} & \frac{\left|w_{i}^{n}-k\right|-\left|\bar{w}_{i}^{n-1}-k\right|}{\Delta t} \varphi_{i}^{n} \frac{h_{i}^{n}+h_{i+1}^{n}}{2} \Delta t \\
= & -\int\left|s_{0}-k\right| \varphi\left(x, t^{1}\right) d x-\int_{0}^{T} \int|\tilde{w}-k| \frac{\partial \varphi}{\partial t} d x d t \\
& +O\left((\Delta t+h)\left(\left\|s_{0}\right\|_{L^{\prime}(\mathbf{R})}+\operatorname{Var}\left(s_{0}\right)\right)\right) \\
& \rightarrow-\int_{0}^{T} \int|w-k| \frac{\partial \varphi}{\partial t} d x d t,
\end{aligned}
$$

since $\varphi(x, 0)=0$. The inequality (3.27) follows from (3.28), (3.32), (3.33), and (3.34), and the weak solution is the unique weak solution satisfying the entropy condition [3], [4]; i.e., the physically relevant solution. Clearly, this also establishes the convergence of the entire sequence of approximate solutions. The following theorem has been proved.

THEOREM. Let the mesh $\delta_{k}^{n}$ at time $t_{k}^{n}$ be subject to the constraints (3.13), but otherwise arbitrary. Let the artificial, weighted diffusivity $g_{i+1 / 2, k}^{n}$ satisfy (3.5) and be bounded above. If $w_{i, k}^{n}$ is the solution of (2.3) corresponding to $\left\{\Delta t_{k}, \delta_{k}^{n}\right\}$ and if $w_{k}$ and $\tilde{w}_{k}$ are the piecewise-bilinear and piecewise-constant extensions of $w_{i, k}^{n}$, then $\left\{w_{k}\right\}$ and $\left\{\tilde{w}_{k}\right\}$ converge in $L^{\infty}\left(0, T ; L^{1}(\mathbf{R})\right)$ to the unique weak solution of $(1.1),(1.7)$ satisfying the entropy condition.

4. Adaptivity Using the Inverse Function. Consider the waterflood problem given by (1.1) with the initial condition

$$
s_{0}(x)= \begin{cases}1, & x<0 \\ 0, & x>0 .\end{cases}
$$

It can be assumed, [1], that $0 \leqslant f(s) \leqslant 1, f(0)=f^{\prime}(0)=f^{\prime}(1)=0$, and $f(1)=1$. Moreover, $f^{\prime}$ can be taken nonnegative and it is bounded. Since the solution is of interest only for $x \geqslant 0$, the analysis of the last section can be applied to difference methods of the form (2.3) to see that approximate solutions converge on $\mathbf{R}^{+} \times[0, T]$ by making a virtual change in $s_{0}$ for $x$ sufficiently negative; in fact, the boundary condition $s(0, t)=1$ can be substituted. 
It can be seen that the entropy condition implies that the relevant solution of (1.1), (4.1) consists of a single shock of height $s^{*}$, where

$$
s^{*} f^{\prime}\left(s^{*}\right)=f\left(s^{*}\right),
$$

moving at the uniform speed $f^{\prime}\left(s^{*}\right)$, a rarefaction between $x=0$ and $x=t f^{\prime}\left(s^{*}\right)$, and the constant zero for $x>t f^{\prime}\left(s^{*}\right)$. If a difference method of the form (2.3) subject to the restraint (3.5) on the artificial diffusivity and the restraint

$$
K_{1} \Delta t \leqslant h_{i}^{n} \leqslant K_{2} \Delta t
$$

is applied to (1.1), (4.1), then convergence in $L^{\infty}\left(0, T ; L^{1}(\mathbf{R})\right)$ is assured as $\Delta t$ tends to zero. A proper interpretation of the difference equation at $x_{0}^{n}$ is given in a mass-conserving form by taking

$$
\frac{w_{0}^{n}-w_{0}^{n-1}}{\Delta t}+\frac{f\left(w_{1}^{n}\right)-f\left(w_{0}^{n}\right)}{h_{1}^{n}}-\frac{g_{1 / 2}^{n}\left(w_{1}^{n}-w_{0}^{n}\right)}{h_{1}^{n}}=\frac{2}{h_{1}^{n}} .
$$

The selection of the grid $\delta^{n}$ can be made in the following fashion [2]. First, select $\delta^{1}=\left\{x_{0}^{1}=0, x_{1}^{1}, \ldots\right\}$, with $x_{i}^{1}=i K_{1} \Delta t$ for small $i$ and set $w_{i}^{1}=0$ for the remaining $i$. Now, it follows from the maximum principle argument used in the proof of Lemma 3.1 that, if $w_{j}^{n-1}$ is decreasing, $w_{i}^{n}$ is also decreasing. Thus, the piecewise-linear interpolant $\hat{w}$ of $w_{i}^{n}$ is decreasing, and its inverse function exists. Moreover, it can be seen easily that $w_{0}^{n} \leqslant 1$. Select the grid $\delta^{n+1}$ tentatively as follows. Let

$$
N_{n}=\frac{2}{\left(K_{1}+K_{2}\right) \Delta t} w_{0}^{n}
$$

and let

$$
\hat{w}^{n}\left(\tilde{x}_{i}^{n+1}\right)=\left(1-\frac{i}{N_{n}}\right) w_{0}^{n}, \quad i=0, \ldots, N_{n}-1
$$

Set

$$
\tilde{x}_{N_{n}}^{n+1}=\max \left(\tilde{x}_{N_{n}-1}^{n+1}+K_{1} \Delta t, \gamma t^{n+1} f^{\prime}\left(s^{*}\right)\right),
$$

where $\gamma$ is bigger than one but less than, say, 1.25 . If necessary, modify $\left\{\tilde{x}_{i}^{n+1}\right\}$ to satisfy (4.3); this can be done by shifting the points or by adding or deleting points. Call the resulting sequence $\delta^{n+1}=\left\{x_{0}^{n+1}, \ldots, x_{M_{n+1}}^{n+1}\right\}$. Then set $w_{M_{n+1}}^{n+1}=0$ and solve for $w_{i}^{n+1}, i=0, \ldots, M_{n+1}-1$.

The algorithm above is essentially the one that was used in [2]. Experimentally, it was found that maintaining the constraint (4.3) placed a sufficiently fine grid around and immediately in front of the location of the approximate shock that it was better not to adjust the grid after each time step, but instead every few steps. In either case convergence takes place. See [2] for results of a number of experiments.

5. Adaptivity Using the Characteristics. Consider the same example as in the last section or the general initial value problem. The characteristic through the point $\left(x_{i}^{n}, t^{n}\right)$ has slope (speed) $f^{\prime}\left(w_{i}^{n}\right)$. Hence, the value $w_{i}^{n}$ would be transported to the point

$$
\tilde{x}_{i}^{n+1}=x_{i}^{n}+f^{\prime}\left(w_{i}^{n}\right) \Delta t
$$

at time $t^{n+1}$, except for shock behavior. Accept some value, say $x_{0}^{n+1}=\tilde{x}_{0}^{n+1}$, as estimated by (5.1). First, reorder to obtain a monotone sequence, then modify this 
sequence to satisfy (4.3), and call the resulting sequence $\delta^{n+1}$. If the example of the last section is treated, cut the sequence off when $x_{i}^{n+1}$ exceeds $\gamma f^{\prime}\left(s^{*}\right) t^{n+1}$. Convergence follows as before.

6. Implicit Upwinding. Return to the example of Section 4. If we set

$$
g_{i+1 / 2}^{n}=F_{i+1 / 2}^{n}
$$

in (2.3) when $h_{i}^{n} \equiv h$, then (2.3) reduces to

$$
\frac{w_{i}^{n}-w_{i}^{n-1}}{\Delta t}+\frac{f\left(w_{i}^{n}\right)-f\left(w_{i-1}^{n}\right)}{h}=0
$$

the standard upwinding scheme, when $f^{\prime} \geqslant 0$. The choice (6.1) does not, in general, satisfy (3.5), and the convergence analysis does not automatically apply. The condition (6.1) does suffice for the maximum principle, and the solution $w_{i}^{n}$ remains decreasing in $x$. Thus, in order that the proof given in Section 3 stand, it is sufficient to show that the term $g_{i+1 / 2}^{n}-G_{i+1}^{n}$ arising in (3.30) be nonnegative. If $w_{i}^{n}>k>$ $w_{i+1}^{n}$, then

$$
\begin{aligned}
g_{i+1 / 2}^{n}-G_{i+1}^{n}= & \left(w_{i}^{n}-w_{i+1}^{n}\right)^{-1}\left(k-w_{i+1}^{n}\right)^{-1} \\
& \times\left[\left(k-w_{i+1}^{n}\right) f\left(w_{i}^{n}\right)-\left(w_{i}^{n}-w_{i+1}^{n}\right) f(k)\right. \\
& \left.\quad+\left(w_{i}^{n}-k\right) f\left(w_{i+1}^{n}\right)\right],
\end{aligned}
$$

which is nonnegative for all decreasing triples $\left(w_{i}^{n}, k, w_{i+1}^{n}\right)$ if and only if $f^{\prime \prime} \geqslant 0$ on $[0,1]$. This condition is never satisfied for the waterflooding problem; however, a minor modification in both the method and the proof allow recapturing both the variable grid and the convergence.

Assume that $f$ is nondecreasing and Lipschitz continuous. Change the definition of the piecewise-constant function $w^{n}$ to be

$$
w^{n}(x)=w_{i}^{n}, \quad x_{i-1}^{n}<x<x_{i}^{n} .
$$

Then, set

$$
\bar{w}_{i}^{n-1}=\frac{1}{h_{i}^{n}} \int_{x_{i-1}^{n}}^{x_{i}^{n}} w^{n-1}(x) d x .
$$

Then, the implicit upwinding difference procedure is given by

$$
\frac{w_{i}^{n}-\bar{w}_{i}^{n-1}}{\Delta t}+\frac{f\left(w_{i}^{n}\right)-f\left(w_{i-1}^{n}\right)}{h_{i}^{n}}=0 .
$$

The analogues of Lemmas 3.1-3.5 are easily proved by essentially the same arguments, again assuming (3.13) to obtain the results corresponding to Lemma 3.5. In particular, it follows that
(a) $\left|w^{n}\right|_{\infty} \leqslant\left\|s_{0}\right\|_{L^{\infty}(\mathbf{R})}$,
(b) $\operatorname{Var}\left(w^{n}\right) \leqslant \operatorname{Var}\left(s_{0}\right)$,
(c) $\sum_{i}\left|\frac{w_{i}^{n}-\bar{w}_{i}^{n-1}}{\Delta t}\right| h_{i}^{n} \leqslant F \operatorname{Var}\left(s_{0}\right)$,
(d) $\sum_{i}\left|w_{i}^{n}\right| h_{i}^{n} \leqslant\left\|s_{0}\right\|_{L^{\prime}(\mathbf{R})}+F t^{n} \operatorname{Var}\left(s_{0}\right)$. 
Again, it follows that subsequences of the corresponding sequences $\left\{w_{k}\right\}$ and $\left\{\tilde{w}_{k}\right\}$ of piecewise-bilinear and -constant interpolants of $\left\{w_{i, k}^{n}\right\}$ converge in $L^{\infty}\left(0, T ; L^{1}(\mathbf{R})\right)$ to a weak solution $w$. The critical step in demonstrating that $w$ is the physically relevant weak solution is the finding of the analogue of (3.28). Here, it follows from the relation $\left(\mu_{i}^{n}=\Delta t / h_{i}^{n}\right)$

$$
\begin{aligned}
\left|w_{i}^{n}-k\right|= & \operatorname{sgn}\left(w_{i}^{n}-k\right) \cdot\left(\bar{w}_{i}^{n-1}-k\right) \\
+\mu_{i}^{n}\left[\operatorname{sgn}\left(w_{i-1}^{n}-k\right)\{\right. & \left.f\left(w_{i-1}^{n}\right)-f(k)\right\} \\
& \left.-\operatorname{sgn}\left(w_{i}^{n}-k\right)\left\{f\left(w_{i}^{n}\right)-f(k)\right\}+R_{i}^{n}\right],
\end{aligned}
$$

where

$$
R_{i}^{n}=\left\{\operatorname{sgn}\left(w_{i}^{n}-k\right)-\operatorname{sgn}\left(w_{i-1}^{n}-k\right)\right\}\left\{f\left(w_{i-1}^{n}\right)-f(k)\right\} \leqslant 0,
$$

that

$$
\begin{aligned}
& \frac{\left|w_{i}^{n}-k\right|-\left|\bar{w}_{i}^{n-1}-k\right|}{\Delta t} \\
& +\frac{\operatorname{sgn}\left(w_{i}^{n}-k\right)\left\{f\left(w_{i}^{n}\right)-f(k)\right\}-\operatorname{sgn}\left(w_{i-1}^{n}\right)\left\{f\left(w_{i-1}^{n}-k\right)-f(k)\right\}}{h_{i}^{n}} \leqslant 0 .
\end{aligned}
$$

Multiplying (6.10) by $\varphi_{i}^{n} h_{i}^{n} \Delta t$ and summing as before leads to seeing that $w$ satisfies (3.27); thus, again convergence is assured in $L^{\infty}\left(0, T ; L^{1}(\mathbf{R})\right)$ for time-dependent, variable grids subject only to the minor restriction (3.13).

The method of selecting $\delta^{n}$ based on using the characteristics in the forward direction in time can be applied for the implicit upwinding scheme for either the initial value problem or for the example given by (1.1), (4.1). Note that multiplication of (6.6) by $h_{i}^{n}$ and summing on $i \geqslant 1$ shows that

$$
\sum_{i=1}^{\infty} w_{i}^{n} h_{i}^{n}=\int_{0}^{\infty} w^{n}(x) d x=\int_{0}^{\infty} w^{n-1}(x) d x+f\left(w_{0}^{n}\right) \Delta t .
$$

Thus, the desired material balance is obtained if the boundary value is set,

$$
w_{0}^{n}=1, \quad n \geqslant 1,
$$

along with the choice $w_{i}^{0}=0, i \geqslant 1$, which is consistent with (6.5). It again follows that $w_{i}^{n}$ decreases in $x$, so that the inverse function procedure for choosing $\delta^{n}$ can be used.

Finally, note that the imposition of (6.12) has the consequence that the procedure is simple algebraically. If $w_{j}^{n-1}, j \geqslant 1$, and $w_{k}^{n}, k=0, \ldots, i-1$, are known, then $w_{i}^{n}$ is the unique root of the equation

$$
w_{i}^{n}+\mu_{i}^{n} f\left(w_{i}^{n}\right)=\gamma_{i}^{n}=\bar{w}_{i}^{n-1}+\mu_{i}^{n} f\left(w_{i-1}^{n}\right) .
$$

The nonlinear equation is particularly easy to solve iteratively when $\delta^{n}$ is picked by means of characteristics, since $w_{i}^{n}$ and $w_{i}^{n-1}$ are nearly equal unless $x_{i}^{n}$ had to be moved from $\tilde{x}_{i}^{n}$.

Department of Mathematics

University of Chicago

Chicago, Illinois 60637

Department of Mathematical Sciences

Rice University

Houston, Texas 77001 
1. J. Douglas, JR., "Simulation of a linear waterflood," Free Boundary Problems, vol. II, Istituto Nazionale di Alta Matematica "Francesco Severi”, Roma, 1980.

2. J. Douglas, JR., B. L. Darlow, M. F. Wheeler \& R. P. Kendall, "Self-adaptive finite element and finite difference methods for one-dimensional, two-phase, immiscible flow," SIAM J. Sci. Statist. Comput. (To appear.)

3. S. N. KRUŽKov, "First order quasilinear equations in several indpendent variables," Math. USSR-Sb., v. 10, 1970, pp. 217-243.

4. P. D. LAX, "Shock waves and entropy," Contributions to Nonlinear Functional Analysis (E. H. Zarantonello, ed.), Academic Press, New York, 1971.

5. A. Y. Le Roux, “A numerical conception of entropy for quasi-linear equations,” Math. Comp., v. 31, 1977, pp. 848-872.

6. M. F. WhEELER, A Self-Adaptive Finite Difference Procedure for One-Dimensional, Two-Phase, Immiscible Flow, Seminar on Numerical Analysis and its Application to Continuum Physics, Coleção Atas, vol. 12, Rio de Janeiro, 1980. 